\title{
A importância do entendimento dos indicadores na tomada de decisão de gestores públicos
}

Danielle Ramos de Miranda Pereira e Marcelo de Rezende Pinto

\section{Introdução}

A existência de uma profunda relação entre educação e desenvolvimento é um pensamento compartilhado entre vários autores, independentemente da forma como interpretam o desenvolvimento, cujo sentido pode variar entre aspectos puramente econômicos e aqueles não somente voltados ao crescimento da produção, mas também ao âmbito social e humano das condições de vida das populações (Schuwartzman, 2003; Barros, Henriques e Mendonça, 2002; Lau et al, 1996).

Nas últimas décadas, o desenvolvimento social tem sido medido por meio de indicadores compostos, também denominados índices, amplamente utilizados pelos gestores públicos como subsídios para a formulação e a avaliação de políticas públicas, e como forma de estreitar a comunicação de suas ações à sociedade, especialmente num contexto em que é crescente a descentralização das ações governamentais e a importância do nível local no planejamento estratégico e na implementação dessas políticas. 
O Índice de Desenvolvimento Humano (IDH) é um indicador criado mediante o esforço do Programa das Nações Unidas para o Desenvolvimento (PNUD) de medir os aspectos sociais e econômicos do desenvolvimento, sintetizando em um único número as dimensões renda, saúde e educação.

Outros índices de desenvolvimento têm surgido com o propósito de medir também as variações de curto prazo, que são extremamente importantes para a avaliação das políticas sociais. Entre esses índices, pode-se citar o Índice Mineiro de Responsabilidade Social (IMRS), que procura captar tais variações, incluindo outras dimensões e indicadores relevantes ao processo de desenvolvimento.

As dimensões "renda do IDH e do IMRS" correspondem ao desenvolvimento puramente econômico. Por sua vez, a dimensão "educação do IDH" inclui aspectos relacionados ao acesso educacional, enquanto essa mesma dimensão do IMRS considera outros fatores além do acesso, tais como qualidade do ensino e recursos disponíveis, frequentemente citados na literatura educacional como indispensáveis ao monitoramento das políticas educacionais (Pereira, 2006; SchuwartzMan, 2003; Cunha, Perez E Aidar, 2001; Castro, 1998; Barros E Mendonça, 1997).

Em face dessa discussão, relativa às diversas formas de se medir dimensões altamente complexas e multifacetadas como renda e educação, algumas questões atinentes ao papel do gestor público parecem emergir: uma vez que existem inúmeras metodologias para mensuração dessas dimensões, como o profissional de gestão pública deve tomar suas decisões? Outra questão nos remete à seguinte indagação: até que ponto a escolha de um indicador pode afetar o diagnóstico, a formulação e o monitoramento de políticas públicas? Não menos importante é a pergunta: como confiar em indicadores, como os de educação, que parecem não conseguir captar toda a complexidade dos fenômenos a que eles se propõem?

Diante dessas inquietações e da complexidade por elas descortinada, surgiu o interesse de elaborar um artigo com os seguintes objetivos: em primeiro lugar, examinar a diferença na relação entre desenvolvimento econômico (expresso em termos de renda) e educação por meio das dimensões de renda e educação, que compõem dois índices de desenvolvimento; em segundo lugar, discutir a importância do entendimento dos indicadores por parte dos gestores públicos; por fim, em terceiro lugar, trazer à baila algumas reflexões acerca da necessidade de se melhorar a qualidade dos indicadores, principalmente de educação, a fim de que possam refletir, de forma mais verdadeira, toda a complexidade dessa dimensão.

Para atingir os objetivos propostos, o trabalho foi estruturado em quatro seções, além desta introdução. Na segunda seção, conduziu-se uma discussão teórica contemplando os temas dos indicadores e suas aplicações. A terceira seção foi acrescentada ao trabalho com o escopo de apresentar os dados referentes à relação entre renda e educação nas regiões administrativas de Minas Gerais. Por fim, na quarta e última seção, as considerações finais do trabalho são apresentadas e discutidas.

\section{Os indicadores e suas aplicações}

Um indicador social pode ser definido como uma medida quantitativa, dotada de significado social substantivo, utilizada para substituir, quantificar ou operacionalizar um conceito social abstrato de interesse teórico para a pesquisa acadêmica ou de 
interesse programático para a formulação de políticas (JANnUZZI, 2002). Os indicadores estabelecem um padrão normativo, por meio do qual é possível construir um diagnóstico para subsidiar a formulação e a avaliação de políticas públicas.

Não obstante a grande utilidade dos indicadores, é essencial salientar que a sua interpretação deve ser acompanhada de uma análise detalhada do fenômeno estudado, uma vez que o alcance dos indicadores é limitado, enquanto tentativa de captar num simples número a complexa realidade social. De acordo com a Organização Mundial de Saúde (Who, 1996) e Jannuzzi (2001), um indicador deve possuir, como propriedades desejáveis, a validade para representar o fenômeno que pretende medir, a confiabilidade de fornecer os mesmos resultados quando calculado em circunstâncias similares, a sensibilidade de refletir mudanças no fenômeno de interesse, a especificidade de refletir mudanças em fenômenos específicos, a relevância para a discussão da agenda da política em questão, o grau de cobertura populacional adequado, a simplicidade para o devido entendimento dos agentes das políticas e do público-alvo dessas políticas, a atualização periódica, a desagregação em termos socioeconômicos e demográficos e, ainda, certa historicidade.

Existem várias tipologias de classificação de indicadores sociais, dependendo, em especial, do fim a que se destinam, da etapa do ciclo de formulação e avaliação de políticas públicas, do seu grau de complexidade e da época em que foram criados. Como instrumentos de avaliação de políticas ou programas sociais, Arretche (1998) e Andrade, Ussan e Klering (2001) classificam os indicadores em: indicadores de eficiência dos recursos empregados, indicadores de eficácia no cumprimento das metas e indicadores da efetividade social dos programas.

Outra tipologia de classificação de indicadores, bastante utilizada na formulação e na avaliação das políticas públicas, divide os indicadores em: indicadores-insumo, indicadores-processo, indicadores-produto e indicadores-impacto (JANNUZZI, 2001). Os indicadores-insumo quantificam os recursos disponibilizados para as políticas públicas, enquanto os indicadores-processo

“Os
indicadores
estabelecem um
padrão
normativo, por
meio do qual é
possível
construir um
diagnóstico
para subsidiar
a formulação e
a avaliação de
políticas
públicas."

medem os esforços de alocação desses recursos para a obtenção de melhorias efetivas no bem-estar social. Por sua vez, os indicadores-produto retratam os resultados efetivos dessas políticas e os indicadores-impacto referem-se aos efeitos sociais alcançados no médio prazo.

Os indicadores também podem ser classificados em função da etapa do ciclo 
de formulação e avaliação de políticas públicas: indicadores para diagnóstico, indicadores para formulação, indicadores para implementação e indicadores para avaliação. Cada etapa desse ciclo, ilustrado na Figura 1, enfatiza o uso de indicadores com determinadas propriedades, dependendo das necessidades intrínsecas das atividades envolvidas.

Segundo Jannuzzi (2005), a primeira etapa do ciclo - a elaboração do diagnóstico da realidade social - inclui retratar a situação social vivenciada pela população, para orientar posteriormente as questões prioritárias a atender, os formatos dos programas a implementar, as estratégias e ações a desenvolver. Para isso, são necessários indicadores de boa confiabilidade, validade e desagregabilidade, contemplando as diversas temáticas da realidade social. O autor salienta que na segunda etapa do ciclo, na formulação de políticas ou programas sociais, os indicadores são selecionados a partir dos objetivos norteadores dos programas definidos como prioritários pela agenda político- social vigente. Para a terceira etapa do ciclo, de implementação e execução dos programas, foi ressaltado que são necessários indicadores de monitoramento com as propriedades da sensibilidade, especificidade e periodicidade, o que constitui um grande problema, especialmente quando se necessita de indicadores em escala municipal.

Por fim, o acompanhamento de programas, que engloba os processos de implementação e avaliação, requer a estruturação de um sistema de indicadores que permita monitorar o dispêndio realizado, o uso operacional dos recursos, a geração de produtos e a percepção dos efeitos sociais dos programas, conforme a lógica insumo-processo-produto-impacto (Cohen e Franco, 2000; JannuzZi, 2005). Dessa forma, no acompanhamento dos programas, a análise da eficiência necessita de indicadores dos esforços e dos recursos alocados, enquanto a análise da efetividade pede indicadores que permitam a vinculação das ações do programa com as mudanças percebidas (ou não) nas

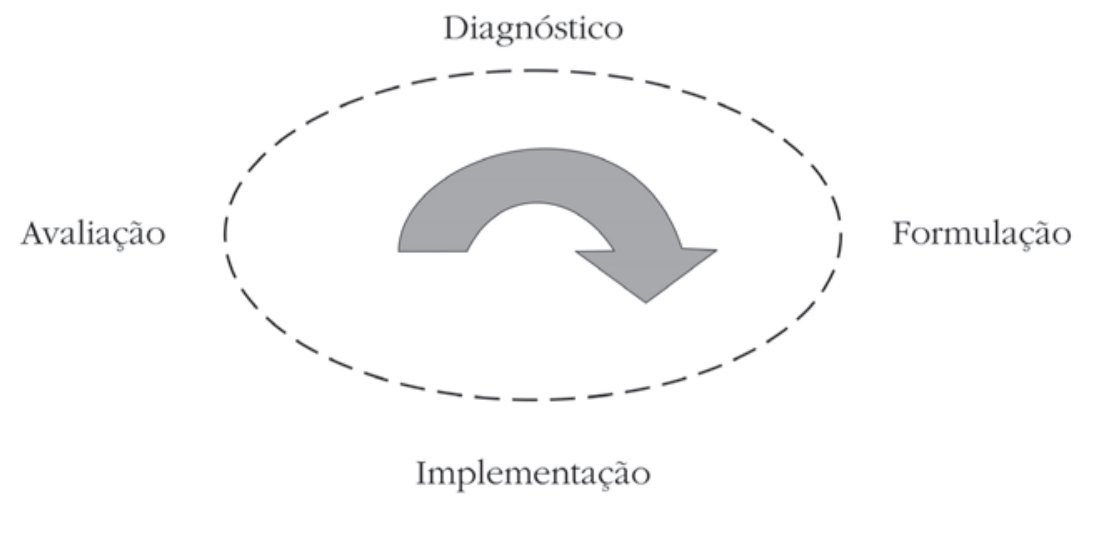

Fonte: Jannuzzi (2005)

Figura 1: Ciclo de formulação e avaliação de políticas ou programas sociais 
condições de vida da população (Roche, 2002; JANNUZZI, 2005).

Quanto ao seu grau de complexidade, os indicadores podem ser classificados em simples ou compostos. Os indicadores compostos, também chamados de indicadores sintéticos ou índices sociais, são um tipo de média estabelecida entre um conjunto de indicadores simples, na tentativa de apresentar, de forma sintética, uma ou mais dimensões da realidade social (JannuZZI, 2001). Esses indicadores sintéticos são instrumentos bastante utilizados na avaliação da gestão pública, permitindo a realização de comparações globais da situação do município e do desempenho da gestão. Em geral, são estabelecidos "pesos" diferenciados para os indicadores que compõem o índice, em função da importância deles na determinação do resultado final. Esses índices podem ter maior aplicação à medida que oferecem ao gestor uma medida-síntese, por exemplo, do desenvolvimento social de municípios onde os programas serão implementados (JANNUZZI, 2005).

Ainda quanto ao grau de complexidade e, também, ao período de sua criação, os indicadores podem ser classificados em três gerações: indicadores de primeira geração, indicadores de segunda geração e indicadores de terceira geração (KAYANO e Caldas, 2002). Os indicadores de primeira geração surgiram no pós-guerra e são indicadores simples, como o Produto Interno Bruto (PIB), criados num contexto em que se preocupava em medir a capacidade de produção de um país, como proxy do seu desenvolvimento (Torres, Ferreira E DINI, 2003). A principal vantagem desse tipo de indicador é a facilidade de entendimento por parte dos gestores públicos e da sociedade, enquanto que a desvantagem em sua utilização está associada ao seu aspecto unidimensional, não captando outras dimensões importantes para o desenvolvimento, como a educação e a saúde.

Por sua vez, os de segunda geração são indicadores compostos, criados na década de 1990, época em que a preocupação central se deslocou do aspecto puramente econômico do desenvolvimento para contemplar, também, o seu âmbito social. Nesse cenário, surgiu o Índice de Desenvolvimento Humano (IDH), que se propôs a enfrentar esse desafio, sintetizando, em um único indicador, dimensões de renda, longevidade e escolaridade.

O IDH foi idealizado pelo Programa das Nações Unidas para o Desenvolvimento (PNUD), para servir de base empírica aos Relatórios de Desenvolvimento Humano, responsáveis por monitorar o processo de desenvolvimento mundial ao longo da década de 1990 (PNUD, 2000; FJP, 2006). Esse índice, que foi fruto de um longo processo de consenso entre pesquisadores, incorpora o PIB per capita na dimensão renda, a esperança de vida ao nascer na dimensão da longevidade, e os indicadores "taxa de alfabetização da população com 15 ou mais anos de idade" e "taxa bruta de matrícula nos níveis de ensino primário, médio e superior" na composição da dimensão de escolaridade (PNUD, Ipea e FJP, 2003).

Entre as principais limitações da utilização do IDH, pode-se destacar a falta de sensibilidade para medidas de curto prazo e para temas de ações puramente municipais (Torres, Ferreira e Dini, 2003; Kayano e Caldas, 2002). Esses mesmos autores ressaltam que a sensibilidade de um indicador, para medir variações de curto prazo, é importante à medida que se pretende avaliar os resultados de políticas e valorizar as ações ligadas à forma de gestão. 
O Índice Paulista de Responsabilidade Social (IPRS) e o Índice Mineiro de Responsabilidade Social (IMRS) são exemplos de indicadores de terceira geração. O IPRS foi elaborado pela fundação Seade, por solicitação da Assembleia Legislativa de São Paulo, para subsidiar a formulação e a avaliação das políticas públicas dos municípios do Estado de São Paulo (Demarco, 2007).

O Índice Mineiro de Responsabilidade Social (IMRS) - que contempla as dimensões renda, saúde, educação, segurança pública, gestão, habitação e meio ambiente, cultura, desporto e lazer - foi desenvolvido para os municípios de Minas Gerais, na tentativa de criar um instrumento mais eficaz para avaliar a situação do desenvolvimento municipal, a atuação da gestão pública e as iniciativas vinculadas à participação nas decisões do município (FJP, 2005).

\section{As dimensões de renda e educação do IDHM e do IMRS}

Esta seção descreve a composição das dimensões "renda e educação do IDHM e do IMRS", enfatizando os fatores educacionais associados ao monitoramento das políticas públicas educacionais. A dimensão "renda do IDH" é composta pelo indicador PIB per capita. Por sua vez, a dimensão "renda do IMRS" inclui indicadores de recursos familiares, de recursos do setor produtivo e da gestão municipal. Os recursos do setor produtivo foram incluídos na dimensão renda do IMRS, porque a Fundação João Pinheiro (FJP, 2005) considera que tais recursos podem ser revertidos em benefício da população.

No tema da gestão municipal, referente à dimensão renda, o IMRS mede os gastos per capita com promoção das atividades agropecuárias e do desenvolvimento econômico, além de considerar a existência e o funcionamento de conselhos nesses setores. Conforme destaca a fundação responsável pela elaboração do índice, a dimensão renda do IMRS é bastante consistente, apresentando elevada correlação com o índice de renda do IDH, em 2000.

Passando à descrição da dimensão "educação", é oportuno destacar que, atualmente, a avaliação do sistema educacional tem procurado contemplar, além do acesso, questões relacionadas à qualidade do ensino e à eficiência no uso dos recursos disponíveis. A qualidade do ensino é geralmente medida por meio de testes padronizados de resultado ou desempenho, cujo modelo teórico está ilustrado na Figura 2. De acordo com essa figura, o modelo teórico "insumo-processo-produto" dos possíveis determinantes do desempenho educacional divide os recursos (insumos) e os processos de aprendizagem em nível de aluno, classe, escola e comunidade.

Os estudos em avaliação educacional têm conseguido demonstrar que a quantidade de recursos financeiros, a infraestrutura das escolas (sala de aula adequada, existência de biblioteca, laboratório e quadra esportiva), a quantidade de professores e seu nível educacional são fatores escolares determinantes do desempenho educacional (Willms e Somers, 1999; Barbosa e Fernandes, 2001; Albernaz, Ferreira e Franco, 2002; SAEB, 2004).

Com relação aos processos de aprendizagem, se, por um lado, é reconhecida a dificuldade de mensurá-los; por outro, não se pode negar a relevância de tais processos para o desempenho dos alunos e, consequentemente, para a qualidade do ensino (LeE e BryK, 1989; WiLlms, 1992 e 2000; Willms e Somers, 1999; Hanusher, 2002 e 2003). Nesse sentido, o relatório Saeb (2004) enfatiza a implantação de conselhos escolares efetivamente atuantes, como forma de alcançar um 


\begin{tabular}{|l|}
\hline Nível comunitário \\
Tamanho da comunidade \\
Nível socioeconômico da \\
comunidade \\
Gastos por aluno \\
\hline Nível da escola \\
Tamanho da escola \\
Gastos por aluno \\
Composição da escola \\
Características do diretor \\
Idade e aparência da construção \\
Acesso a recursos comunitários \\
\hline Nível da classe \\
Tamanho da classe \\
Características dos professores \\
Aparência da sala \\
Recursos instrucionais \\
Composição da classe \\
\hline Inputs de aluno \\
Sexo e raça \\
Eendimento prévio \\
Ocupação dos pais \\
\hline
\end{tabular}

\section{Processo- Nível comunitário}

Segregação entre escolas Relação entre escola e comunidade

\section{Processo- Nível escola}

Liderança instrucional do diretor Clima disciplinar

Tracking

Relacionamento entre pais e escola

\section{Processo- Nível classe}

Condições de trabalho

Senso de eficácia do professor Moral do professor

Clima disciplinar

Agrupamento por habilidade (tracking)

\section{Processo- Nível aluno}

Qualidade de vida na escola Senso de eficácia do aluno Atitudes do aluno perante a escola

Fonte: Adaptado de Willms (1992)

\section{Figura 2: Modelo insumo-processo-produto para os resultados educacionais}

processo escolar que conduz a resultados mais bem sucedidos.

Em consonância com os fatores geralmente associados à avaliação do sistema educacional e ao monitoramento das políticas públicas educacionais, conforme descrito acima, a dimensão "educação do IMRS" inclui indicadores de acesso à educação, de adequação desse acesso e da qualidade do ensino, além de indicadores relacionados à gestão municipal; enquanto a dimensão "educação do IDH" contempla apenas indicadores relacionados ao acesso educacional, tais como a taxa de alfabetização da população de 15 ou mais anos de idade e a taxa bruta de matrícula nos níveis de ensino primário, médio e superior.

Para a avaliação do acesso, o IMRS considera as taxas de atendimento das crianças de quatro a seis anos, das crianças de sete a 14, dos adolescentes de 15 a 17 e das crianças e adolescentes de sete a 17 anos. Partindo-se do pressuposto de que acesso adequado significa que as pessoas de 15 anos tenham concluído o ensino fundamental e que as de 18 tenham concluído o ensino médio; na dimensão de adequação do acesso - adequação série-idade -, foram incluídas as matrículas da população de 15 ou mais anos de idade 
no ensino fundamental e da população de 18 ou mais anos de idade no ensino médio, como proporção do total das matrículas nesses dois níveis de ensino.

Quanto à qualidade do ensino, foram gerados indicadores a partir dos resultados dos exames de língua portuguesa e de matemática do Sistema Mineiro de Avaliação da Educação Pública (Simave), aplicados aos alunos da $8^{a}$ série do Ensino Fundamental e da $3^{a}$ série do Ensino Médio das escolas estaduais; além de outros, relacionados ao acesso dos alunos a laboratórios de informática e à Internet. No tema da gestão municipal em educação, são medidos ou mensurados os gastos per capita em educação e a participação percentual desses gastos no orçamento municipal. Nesse tema, foi incluído ainda o indicador relacionado à existência de conselho atuante nas escolas.

Cabe lembrar ainda que o IDH em nível municipal (IDHM) foi adaptado metodologicamente para melhor captar a realidade, uma vez que os municípios, diferentemente dos países, não devem ser considerados razoavelmente fechados do ponto de vista demográfico. Assim, para garantir melhor comparabilidade entre os municípios e uma representação mais fiel dos aspectos da vida humana, o IDHM foi adaptado, substituindo - na dimensão "renda" - o PIB per capita pela renda familiar per capita e - na dimensão "educação" - a taxa de matrícula pela taxa de frequência à escola (FJP, 2006).

Relação entre renda e educação nas regiões administrativas de Minas Gerais, a partir das dimensões correspondentes no IDHM e no IMRS

\section{Dados e metodologia}

Os dados utilizados neste artigo se referem ao Índice Mineiro de Responsabi- lidade Social (FJP, 2005) e ao Índice de Desenvolvimento Humano Municipal (PNUD, IPEA e FJP, 2003); mais precisamente, às suas dimensões de renda e educação para os municípios do Estado de Minas Gerais, em 2000, disponíveis em bases de dados. Os municípios foram agrupados levando-se em consideração as 10 regiões administrativas do Estado de Minas Gerais, conforme Figura 3.

A relação entre renda e educação dos municípios agrupados nas 10 regiões administrativas de Minas Gerais (Norte de Minas, Nordeste de Minas, Jequitinhonha/Mucuri, Rio Doce, Mata, Central Sul de Minas, Centro-Oeste de Minas, Alto Paranaíba e Triângulo), ilustradas na Figura 3, foi verificada por meio desses dois índices, ajustando-se um modelo de regressão linear simples. A análise de regressão linear simples consiste em ajustar um modelo para estimar a associação linear entre duas variáveis (HAIR et al, 2005). Essa associação é mensurada pela estatística $r$, que resume a intensidade e a direção da associação entre as duas variáveis. Quanto mais próximo de 1, mais forte é a relação. Caso r seja 0 , não há relação entre as duas variáveis. Ou seja, foi verificada a relação entre as variáveis "renda e educação" para todos os municípios do estado, agrupados por região.

\section{Análise dos resultados}

A Tabela 1 mostra as médias referentes às dimensões de renda e de educação dos índices IMRS e IDHM, por região administrativa do Estado de Minas Gerais, em 2000. As médias da dimensão renda variaram entre 0,35 e 0,64 no IMRS, e entre 0,52 e 0,70 no IDHM; enquanto o intervalo das médias da dimensão educação foi de 0,66- 1,00 no IMRS e de 0,70-0,84 no IDHM. As regiões Norte de Minas e Jequitinhonha/Mucuri apresentaram as 


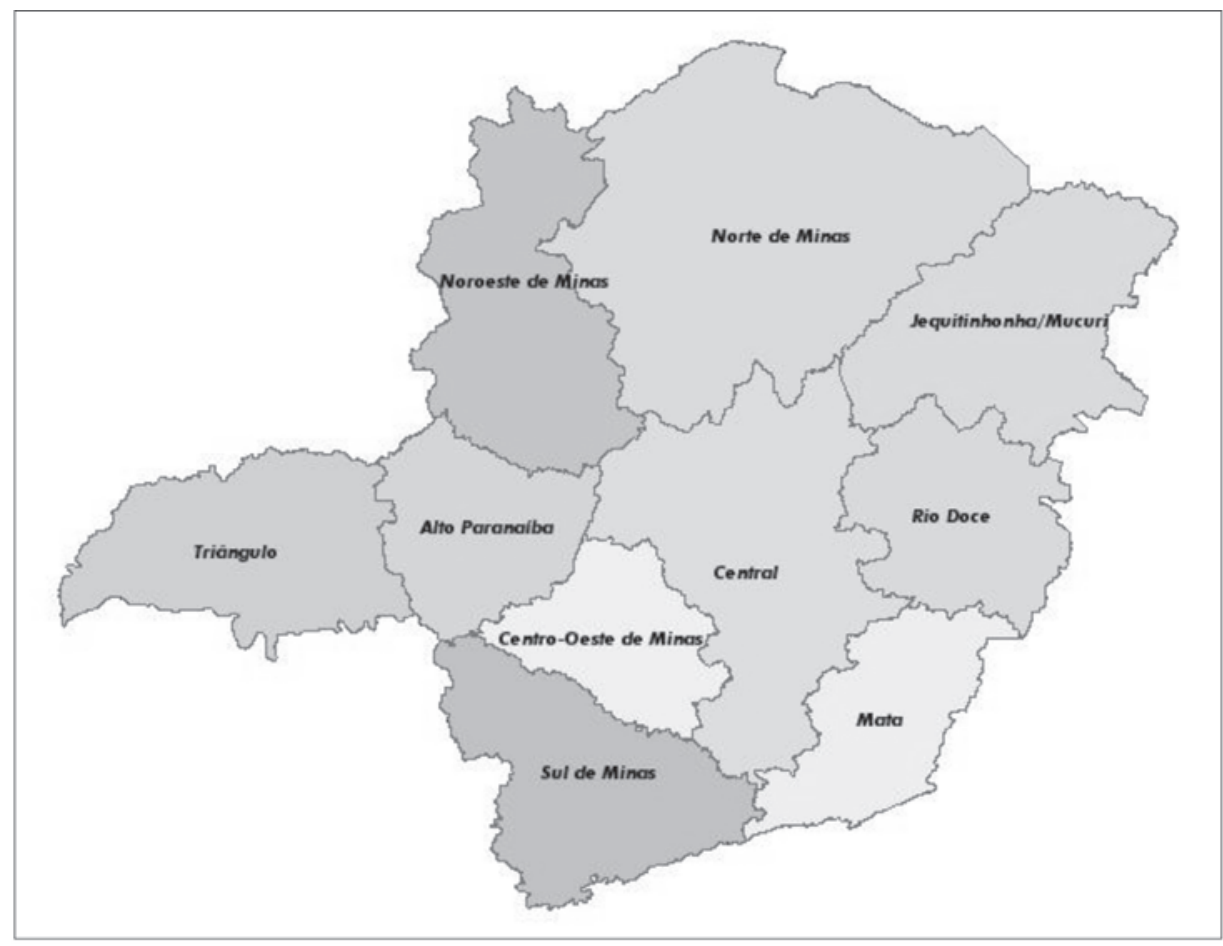

Fonte: Secretaria de Desenvolvimento Econômico de Minas Gerais (2008)

Figura 3: Regiões administrativas do Estado de Minas Gerais

Tabela 1: Média para as dimensões renda e educação, de acordo com o IMRS e IDHM, por região administrativa de Minas Gerais, 2000

\begin{tabular}{l|c|c|c|c}
\hline \multirow{2}{*}{ Nome região } & \multicolumn{2}{|c|}{ Média_IMRS } & \multicolumn{2}{c}{ Média_IDHM } \\
\cline { 2 - 5 } & Renda & Educação & Renda & Educação \\
\hline Norte de Minas & 0,35 & 0,66 & 0,52 & 0,73 \\
\hline Nordeste de Minas & 0,51 & 0,69 & 0,65 & 0,82 \\
\hline Jequitinhonha/Mucuri & 0,37 & 0,66 & 0,54 & 0,70 \\
\hline Rio Doce & 0,44 & 0,67 & 0,59 & 0,75 \\
\hline Mata & 0,47 & 0,66 & 0,63 & 0,79 \\
\hline Central & 0,51 & 1,00 & 0,62 & 0,83 \\
\hline Sul de Minas & 0,54 & 0,67 & 0,68 & 0,82 \\
\hline Centro Oeste de Minas & 0,55 & 0,68 & 0,68 & 0,82 \\
\hline Alto Paranaíba & 0,58 & 0,68 & 0,70 & 0,83 \\
\hline Triângulo & 0,64 & 0,70 & 0,70 & 0,84 \\
\hline
\end{tabular}

Fonte: Elaborada pelos autores 
menores médias na dimensão renda, tanto no IMRS ( 0,35 e 0,37 respectivamente) quanto no IDHM $(0,52$ e 0,54$)$. Por outro lado, as regiões Triângulo e Alto Paranaíba possuem as maiores médias na dimensão renda do IMRS $(0,64$ e 0,58) e do IDHM (0,70 em ambas as regiões). Quanto à dimensão educação, as regiões Norte de Minas, Jequitinhonha/Mucuri e Mata são responsáveis pelos menores índices de educação no estado, de acordo com o IMRS $(0,66)$; enquanto os maiores índices para essa dimensão foram registrados nas regiões Central $(1,00)$ e Triângulo $(0,70)$. Por sua vez, a dimensão educação do IDHM registra os menores valores nas regiões Jequitinhonha/Mucuri $(0,70)$ e Norte $(0,73)$, e o maior valor na região Triângulo $(0,84)$.

A Tabela 2 apresenta os resultados da associação linear entre as dimensões renda do IMRS e do IDHM, para as 10 regiões administrativas do Estado de Minas Gerais, em 2000. Esses resultados confirmam a associação significativa $($ sig $<0,05)$ entre as dimensões de renda desses dois índices em todas as regiões administrativas do Estado, conforme havia destacado a Fundação João Pinheiro na ocasião da criação do IMRS. Vale ressaltar ainda que, somente no Triângulo Mineiro, a associação linear entre as dimensões de renda foi inferior a $50 \%$.

De acordo com a Tabela 3, não se pode afirmar o mesmo quando se verifica a associação linear entre as dimensões de educação do IMRS e do IDHM, uma vez que somente nas regiões Mata e Sul de Minas essa associação foi significativa $($ sig<0,05). Contudo, o grau com que as dimensões de educação estão associadas nessas duas regiões não ultrapassa 35\%.

Diante dos resultados descritos acima, quatro modelos de regressão foram ajustados para as 10 regiões administrativas estudadas. De início, "fixada" a variável renda do IMRS como dependente, foram conduzidos dois tipos de análise de regressão: um com a variável independente "educação do IDHM" e outro com a variável independente "educação do IMRS", conforme mostra a Tabela 4. Quando se considera a variável educação do IDHM, as associações lineares entre renda e educação foram significativas em todas as regiões administrativas, variando em intensidade entre 0,40 (no Alto Paranaíba) e 0,81 (na região Central).

Por outro lado, quando a variável independente é a educação do IMRS, a associação entre renda e educação foi significativa apenas nas regiões Mata $($ sig $=0,00)$ e Central $(\operatorname{sig}=0,01)$. Vale destacar que essas associações apresentam intensidade inferior a 40\%, valor mínimo encontrado quando a variável independente é a educação do IDHM.

Em seguida, fixando a variável renda do IDHM como dependente, foram conduzidos outros dois tipos de análise de regressão: um com a variável independente educação do IDHM e outro com a variável independente educação do IMRS, de acordo com as informações apresentadas na Tabela 5.

Se a variável dependente for a educação do IDHM, as associações lineares entre renda e educação foram significativas e relativamente altas em todas as regiões administrativas, variando em intensidade entre 0,51 (no Nordeste de Minas e no Triângulo) e 0,81 (na região Central). Quando a variável independente é a educação do IMRS, a associação entre renda e educação foi significativa apenas na região da Mata (sig=0,04), apresentando uma intensidade de $17 \%$. 
Tabela 2: Associação linear entre as dimensões de renda do IMRS e IDHM, por região administrativa de Minas Gerais, 2000

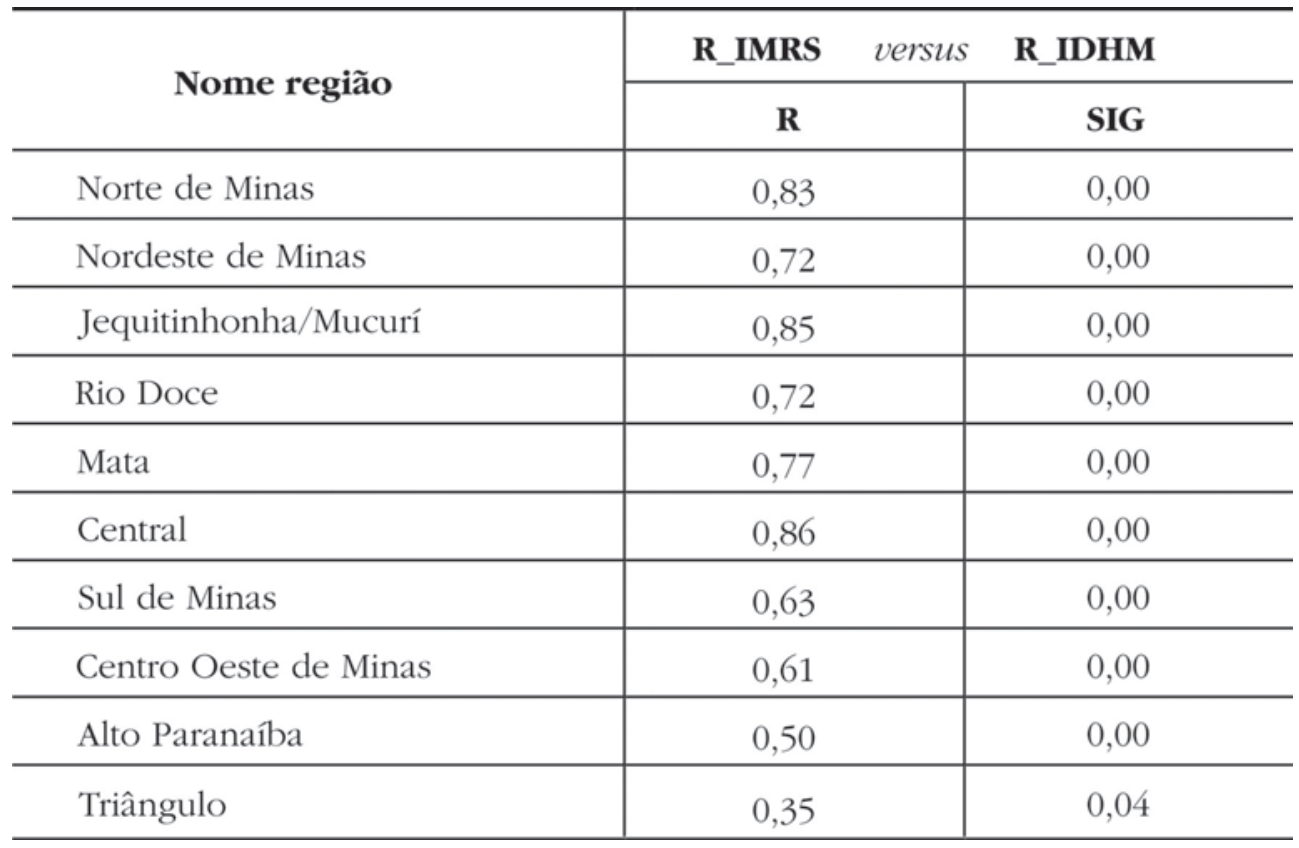

Fonte: Elaborada pelos autores

Tabela 3: Associação linear entre as dimensões de educação do IMRS e IDHM, por região administrativa de Minas Gerais, 2000

\begin{tabular}{l|c|c}
\hline \multirow{2}{*}{ Nome região } & E_IMRS versus & E_IDHM \\
\cline { 2 - 3 } & $\mathbf{R}$ & SIG \\
\hline Norte de Minas & 0,15 & 0,16 \\
\hline Nordeste de Minas & 0,09 & 0,71 \\
\hline Jequitinhonha/Mucuri & 0,18 & 0,14 \\
\hline Rio Doce & 0,10 & 0,32 \\
\hline Mata & 0,32 & 0,00 \\
\hline Central & 0,05 & 0,53 \\
\hline Sul de Minas & 0,15 & 0,05 \\
\hline Centro Oeste de Minas & 0,22 & 0,10 \\
\hline Alto Paranaíba & 0,26 & 0,16 \\
\hline Triângulo & 0,27 & 0,11 \\
\hline
\end{tabular}

Fonte: Elaborada pelos autores 
Tabela 4: Associação linear entre renda do IMRS e educação, por região administrativa de Minas Gerais, 2000

\begin{tabular}{|c|c|c|c|c|}
\hline \multirow{2}{*}{ Nome região } & \multicolumn{2}{|c|}{ R_IMRS versus $\mathbf{E} \_I D H M$} & \multicolumn{2}{|c|}{ R_IMRS versus E_IMRS } \\
\hline & $\mathbf{R}$ & $\mathbf{R}$ & $\mathbf{R}$ & SIG \\
\hline Norte de Minas & 0,72 & 0,04 & 0,04 & 0,71 \\
\hline Nordeste de Minas & 0,52 & 0,16 & 0,16 & 0,52 \\
\hline Jequitinhonha/Mucuri & 0,60 & 0,17 & 0,17 & 0,16 \\
\hline Rio Doce & 0,64 & 0,04 & 0,04 & 0,73 \\
\hline Mata & 0,65 & 0,31 & 0,31 & 0,00 \\
\hline Central & 0,81 & 0,20 & 0,20 & 0,01 \\
\hline Sul de Minas & 0,52 & 0,06 & 0,06 & 0,43 \\
\hline Centro Oeste de Minas & 0,61 & 0,06 & 0,06 & 0,65 \\
\hline Alto Paranaíba & 0,40 & 0,28 & 0,28 & 0,14 \\
\hline Triângulo & 0,74 & 0,16 & 0,16 & 0,37 \\
\hline
\end{tabular}

Fonte: Elaborada pelos autores

Tabela 5: Associação linear entre renda do IDHM e educação, por região administrativa de Minas Gerais, 2000

\begin{tabular}{l|c|c|c|c}
\hline \multirow{2}{*}{ Nome região } & \multicolumn{2}{|c|}{ R_IDHM } & versus $\mathbf{E}$ IIDHM & \multicolumn{2}{c}{ R_IDHM } & versus E_IMRS \\
\cline { 2 - 5 } & $\mathbf{R}$ & SIG & $\mathbf{R}$ & SIG \\
\hline Norte de Minas & 0,71 & 0,00 & 0,00 & 0,98 \\
\hline Nordeste de Minas & 0,51 & 0,03 & 0,14 & 0,56 \\
\hline Jequitinhonha/Mucuri & 0,60 & 0,00 & 0,11 & 0,39 \\
\hline Rio Doce & 0,75 & 0,00 & 0,09 & 0,36 \\
\hline Mata & 0,75 & 0,00 & 0,17 & 0,04 \\
\hline Central & 0,81 & 0,00 & 0,11 & 0,16 \\
\hline Sul de Minas & 0,62 & 0,00 & 0,12 & 0,15 \\
\hline Centro Oeste de Minas & 0,74 & 0,00 & 0,15 & 0,27 \\
\hline Alto Paranaíba & 0,54 & 0,00 & 0,18 & 0,32 \\
\hline Triângulo & 0,51 & 0,00 & 0,27 & 0,12 \\
\hline
\end{tabular}

Fonte: Elaborada pelos autores 


\section{Considerações finais}

De acordo com os resultados obtidos nos modelos de regressão ajustados, a associação entre renda e educação, quando se considera a dimensão educação do IMRS, pode não ser significativa ou possuir intensidade bem menor. É possível perceber uma diferença nos resultados da associação entre renda e educação quando se utilizam indicadores diferentes. Essa constatação parece sinalizar, pelo menos, duas questões relevantes: primeiro, a composição dos indicadores tem influência direta em seus resultados; segundo, isso indica que a dimensão educação deve incluir outros aspectos além do acesso, como procurou fazer o IMRS, ao considerar também os fatores educacionais relacionados à adequação desse acesso, à qualidade do ensino e aos recursos aplicados no sistema educacional. No entanto, os índices de educação ainda não contemplam, de forma significativa, os fatores associados ao processo ou ao capital social que, embora difíceis de serem quantificados, são extremamente importantes para o monitoramento das políticas públicas educacionais.

A partir do exposto nas seções anteriores, é possível fazer considerações finais para este estudo, enfatizando o alcance dos objetivos propostos. Com relação ao primeiro e segundo objetivos, foram verificados resultados não coincidentes para a relação entre renda e educação, a partir do IDHM e do IMRS; pois enquanto essa relação no IDHM era significativa e forte, no IMRS ela se mostrou não significativa ou bem menos intensa. Essa constatação é de importância para o administrador público, pois the atribui uma grande responsabilidade ao selecionar os indicadores que balizarão suas ações, tanto na formulação quanto no acompanhamento e na avaliação das políticas públicas. A discordância nos resultados e/ou a pouca habilidade para "ler" quais aspectos compõem o indicador podem levar o gestor público a tomar decisões equivocadas ou não coerentes com a sua realidade.

Com relação ao terceiro objetivo estabelecido, cabe ressaltar uma noção que

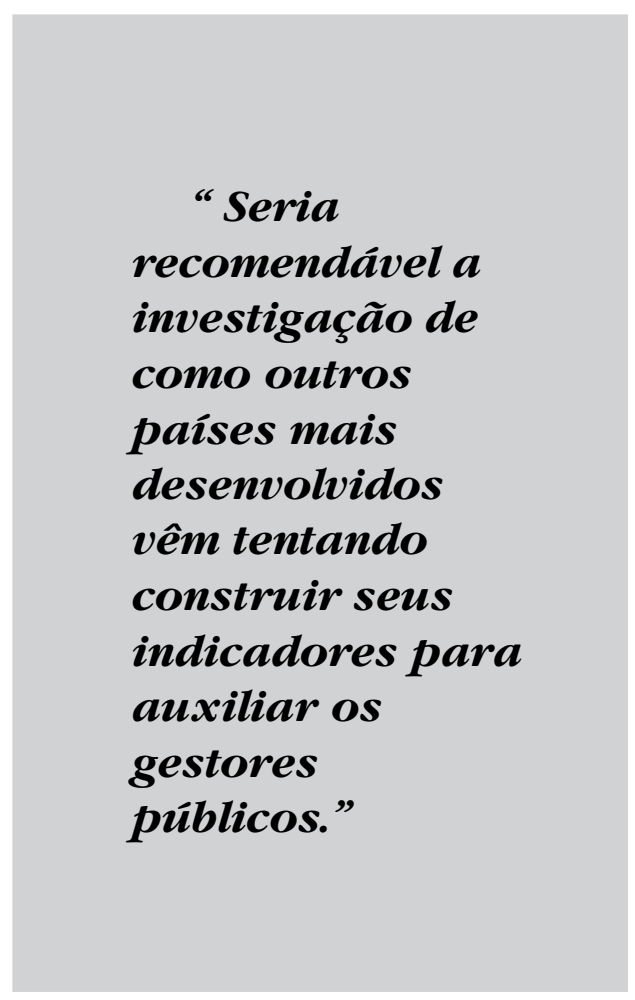

deve nortear qualquer discussão envolvendo o tema dos indicadores: o limite do seu alcance ao captar a complexidade da vida social. Isso significa que a tentativa de mensurar, padronizar, equalizar situações e contextos que são extremamente diferentes, multifacetados e, principalmente, construídos histórica e socialmente não é tarefa das mais simples. Em se tratando de indicadores de educação, essa noção torna-se ainda mais correta, pois é amplamente 
sabido que eles parecem não conseguir captar toda a complexidade dos fenômenos, apesar de todo um esforço canalizado no sentido de se incluir questões mais amplas na composição desses indicadores. Assim, a discussão levantada por este trabalho conduz a uma certeza inexorável: os indicadores sociais de educação devem caminhar no sentido de incorporar variáveis referentes ao que vários autores denominam processo ou capital social, contemplando questões associadas ao clima disciplinar, à liderança e ao relacionamento entre os atores desse processo. Questões atinentes ao clima organizacional na escola e à participação dessa na vida da comunidade à qual está inserida são relevantes também. Em toda essa discussão, não se poderia deixar de registrar a dificuldade adjacente à mensuração dessas relações. Embora os indicadores de terceira geração, como o IMRS, já tenham tentado incluir essas relações, também é coerente afirmar que muito ainda precisa ser feito para se ter indicadores de educação que retratem com maior validade a situação dos municípios nessa questão.

Dessa forma, esta parte do trabalho busca refletir sobre o papel dos gestores públicos no tocante a todas as questões colocadas anteriormente. Isso reforça a necessidade de os administradores públicos procurarem conhecer com detalhes a forma como os indicadores foram construídos, seu alcance e, principalmente, suas limitações. No mesmo sentido, devem comparar os resultados de vários desses indicadores, a fim de verificar qual ou quais deles refletem mais adequadamente sua realidade. Para tanto, as insti- tuições e órgãos de pesquisa estaduais e federais devem investir na construção de indicadores cada vez mais elaborados, o que incentivaria os gestores públicos a utilizarem esses indicadores como importante ferramenta de gestão.

Nesse ponto, cumpre salientar algumas limitações do trabalho. $\mathrm{O}$ artigo se limitou à análise de apenas um dos estados da Federação - estado esse que tem características próprias e diferentes em relação ao restante do Brasil. Ainda se pode considerar como limitação do trabalho o fato de se concentrar somente na relação entre os indicadores de renda e de educação, extraídos de dois índices de desenvolvimento.

Por fim, a partir dessas limitações, podem ser propostas algumas sugestões para futuros trabalhos. Outros estudos poderiam ser conduzidos buscando-se estabelecer novas relações entre outros indicadores ligados às condições de saúde, habitação, etc. Novas pesquisas poderiam investigar estados brasileiros com diferentes realidades sociais por meio de outros índices, a fim de se aprofundar nas questões abordadas neste artigo. Também seria interessante a condução de estudos comparativos ao longo do tempo para melhor verificar mudanças no perfil dos indicadores. Porém, a grande sugestão para futuras pesquisas é a de propor novas metodologias para a construção de indicadores que possam retratar mais fielmente os fenômenos. Assim, seria recomendável a investigação de como outros países mais desenvolvidos vêm tentando construir seus indicadores para auxiliar os gestores públicos.

(Artigo recebido em maio de 2012. Versão final em agosto de 2012). 


\section{Referências bibliográficas}

Albernaz, A.; Ferreira, F.H.g.; Franco, C. Qualidade e equidade no ensino fundamental brasileiro. Pesquisa e planejamento econômico. Rio de Janeiro, v. 32, n. 3, p.453-476, dez.2002. Instituto de Pesquisa Econômica Aplicada.

Andrade, J. A.; Ussan, J. L. M; Klering, L. R. A Avaliação de Políticas Públicas em Análise: o caso do Sistema Nacional de Avaliação da Educação Básica - SAEB. In: Encontro Anual da Associação Nacional dos Programas de Pós-graduação em Administração, 25 Anais... Campinas: ANPAD, 2001.p. 1-14. CD-ROM.

Arretche, M. T. Tendências no Estudo sobre Avaliação. In: RICO, Elizabeth Melo (org.) Avaliação de políticas sociais: uma questão em debate. São Paulo: Cortez/Iee, 1998. Barbosa, M.E.F.; Fernandes, C. A escola brasileira faz diferença? Uma investigação dos efeitos da escola na proficiência em matemática dos alunos da $4^{a}$ série. In: FRANCO, C. (org.). Avaliação, ciclos e promoção na Educação. Porto Alegre: ARTMED, 2001.

Barros, R. P; MendonçA, R. Investimentos em Educação e Desenvolvimento Econômico. Texto para Discussão n $n^{\circ}$ 525. Rio de Janeiro: IPEA, 1997.

Barros, R. P; Henriques, R.; Mendonça, R. Pelo Fim das Décadas Perdidas: Educação e Desenvolvimento Sustentado no Brasil. Texto para Discussão $n^{\circ}$ 857. Rio de Janeiro: IPEA, 2002.

Castro, M. H. Avaliação do Sistema Educacional Brasileiro: tendências e perspectivas. Brasília: MEC/INEP, 1998.

Cohen, E.; Franco, R. Avaliação de projetos sociais. Petrópolis: Vozes, 2000.

Cunha, J. M. P.; Perez, J. R.; Aidar, T. Proposta Metodológica de Elaboração de Indicador Educacional Sintético para os municípios. Revista Brasileira de Estudos de População, v.18, n.1/2, p. 131-159, 2001.

Demarco, D. J. Educação e Desenvolvimento. O Índice Paulista de Responsabilidade Social nos Municípios do Noroeste Paulista. 2007. Tese (Doutorado em Educação) - USP, São Paulo.

Fundação João PINHeIro (FJP). Metodologia de Cálculo dos Indicadores e Índices de Desenvolvimento Humano. Atlas do Desenvolvimento Humano da Região Metropolitana de Belo Horizonte. Belo Horizonte: Fundação João Pinheiro, 2006. CD-ROM.

. Indice Mineiro de Responsabilidade Social. Belo Horizonte: Fundação João

Pinheiro, 2005. CD-ROM.

Fundação João Pinheiro (FjP) E Instituto De Pesquisas Econômicas Aplicadas (IPEA). Definição e Metodologia de Cálculo dos Indicadores e Índices de Desenvolvimento Humano e Condições de Vida, 1998.

Fundação Seade. Metodologia do IPRS 2004. São Paulo, 2004.

Hair, J. F. Jr.; Anderson, R. E.; Tatham, R. L. E Black, W. C. Análise Multivariada de Dados. $5^{a}$ edição. Porto Alegre: Bookman, 2005.

HanusheK, E. A. The failure of input-based schooling policies. The Economic Journal, v. 113, p.64-98, Feb. 2003. 
Hanusher, E. A. Publicy provided education. In: Auerbach , A. J., Feldstein, M. Handbook of Public Economics. Stanford: Elsevier Science B.V., v. 4, p. 2046-2107, 2002.

Jannuzzi, P. M. Indicadores sociais no Brasil: conceitos, fontes e aplicações. Campinas: Alínea/ PUC-Campinas, 2001.

- Considerações sobre o uso, mau uso e abuso dos indicadores sociais na

formulação e avaliação de políticas públicas municipais. Revista de Administração Pública, Rio de Janeiro, v. 36, n.1, p.51-72, Jan. /Fev. 2002.

. Indicadores para diagnóstico, monitoramento e avaliação de programas

sociais no Brasil. Revista do Serviço Público, Brasilia, v. 56, n.2, p. 137-160, Abr/Jun 2005.

Kayano, J.; Caldas, E. de L. Indicadores para o Diálogo. GT Indicadores. Plataforma Contrapartes Novib. Série Indicadores, São Paulo, n. 8, p. 1-10, 2002.

LAU, L.J.; Jamison, D.T.; LiU, S; RiUKin, S. Education and economic growth: some cross-sectional evidence. Opportunity foregone: education in Brazil. Washington: BID, p.83-116, 1996.

LEE, V. E.; BRYK, A. S. A multilevel model of the social distribution of high school achievement. Sociology of Education, v.62, p.172-192, 1989.

Pereira, D. R. M. Fatores Associados ao Desempenho Escolar nas Disciplinas de Matemática e de Português no Ensino Fundamental: Uma Perspectiva Longitudinal. 2006. Tese (Doutorado em Demografia) - CEDEPLAR/UFMG, Belo Horizonte.

Programa Das Nações Unidas Para o Desenvolvimento (PNUD). Relatório do desenvolvimento humano 2000. Lisboa: Trinova, 2000.

Programa Das Nações Unidas Para o Desenvolvimento (PNUD), Instituto De Pesquisas Econômicas Aplicadas (IPEA) E Fundação joão Pinheiro (FJP). Atlas do Desenvolvimento Humano no Brasil, 2003. CD-ROM.

Roche, C. Avaliação de impacto dos trabalhos de ONGs. São Paulo: Cortez, 2002.

SAEB - Sistema Nacional de Avaliação da Educação Básica. Relatório SAEB99. Disponível em: <www.inep.gov.br>.

SCHWARTZMAn, S. Educação e Desenvolvimento: Onde estamos, e para aonde vamos? In: Seminário "Brasil em Desenvolvimento". Rio de Janeiro: Instituto de Economia, Universidade Federal do Rio de Janeiro, 2003.

Secretaria de Estado de Desenvolvimento Econômico de Minas Gerais. Disponível em: www.desenvolvimento.mg.gov.br.

Torres, H. G.; Ferreira, M. P.; Dini, N. P. Indicadores sociais: por que construir novos indicadores como o IPRS. São Paulo em Perspectiva, v. 17, n. 3-4, p. 80-90, jul.-dez. 2003. Willms, J.D. Monitoring school performance: a non-technical guide for educational administrators. Lewes: Falmer press, 1992.

Willms, J. D.; Somers, M-A. Schooling outcomes in Latin America. A report for UNESCO, 1999.

WiLLMS, J. D. Monitoring school performance for "standards-based reform". Evaluation and Research in Education, v.14, n. 3 e 4, p. 237-253, 2000.

World Health Organization (WHO). Catalogue of health indicators. Genebra, 1996. 


\section{Resumo - Resumen - Abstract}

\section{A importância do entendimento dos indicadores na tomada de decisão de gestores públicos}

Danielle Ramos de Miranda Pereira e Marcelo de Rezende Pinto

$\mathrm{Na}$ literatura, existe concordância sobre a relação entre educação e desenvolvimento nos seus diversos sentidos. A partir dessa premissa, o artigo pretende examinar a diferença na relação entre desenvolvimento econômico (expresso em termos de renda) e educação, por meio das dimensões "renda e educação" de dois índices de desenvolvimento: o IDHM e o IMRS. Essa diferença foi estudada em relação às 10 regiões administrativas de Minas Gerais, exemplificando como a decisão dos gestores públicos pode ser influenciada pela seleção de diferentes indicadores, utilizados na formulação ou na avaliação das políticas públicas. Os resultados apontam que, no IMRS, a relação entre renda e educação pode não ter sido significativa ou ter sido menos intensa que a encontrada, quando se toma como referência o IDHM. Isso pode significar que a dimensão "educação", nos indicadores, precisa incorporar, além das questões de acesso, aquelas relacionadas à qualidade da educação e aos recursos disponíveis.

Palavras-chave: Indicadores sociais; IDHM; IMRS

La importancia de los indicadores en la comprensión de la toma de decisiones de los gerentes públicos

Danielle Ramos de Miranda Pereira y Marcelo de Rezende Pinto

En la literatura se coincide en la relación entre educación y desarrollo en sus diversos sentidos. A partir de esta premisa, el artículo busca examinar las diferencias en la relación entre el desarrollo económico (expresado en términos de ingresos) y la educación, a través de las dimensiones de ingresos y educación de los dos índices de desarrollo: el IMRS e IDHM. Esta diferencia se ha estudiado en las diez regiones administrativas de Minas Gerais (Brasil), que ejemplifica cómo la decisión de los funcionarios públicos puede ser influenciada por la selección de los diferentes indicadores utilizados en el desarrollo o evaluación de políticas públicas. Los resultados muestran que en el IMRS, la relación entre la renta y la educación puede que no haya sido significativa o haya sido menos intensa que la que normalmente se encuentra cuando se toma como referencia el IDHM. Esto puede significar que los indicadores de tamaño en la educación deben incorporar, además de problemas de acceso, los relacionados con la calidad de la educación y los recursos.

Palabras clave: Indicadores sociales; IDHM; IMRS

\section{The importance of understanding indicators in decision making in public} management

Danielle Ramos de Miranda Pereira and Marcelo de Rezende Pinto

In the literature there is agreement with the relationship between education and development in its various senses. From that premise, the paper aims to examine the difference in the relationship between economic development (in terms of income) and education by means of education and income dimensions of two indices of development, the MHDI and MSRI. This difference has been studied for the ten administrative regions of Minas Gerais, exemplifying how the decision of public managers may be influenced by the selection of different indicators used in the formulation or evaluation of public policies. The results indicate that the MSRI the relationship between income and education was not significant or less intensive than the generally found 
when taking as reference the MHDI. This may mean that the size indicators in education need to incorporate, in addition to issues of access, those related to the quality of education and resources.

Keywords: Social indicators; MHDI; MSRI

Danielle Ramos de Miranda Pereira

Doutora em demografia pelo Centro de Desenvolvimento e Planejamento Regional da Universidade Federal de Minas Gerais (CEDEPLAR/UFMG). É pesquisadora em Ciência e Tecnologia da Fundação João Pinheiro (FJP). Contato: danielle.pereira@fjp.mg.gov.br

Marcelo de Rezende Pinto

Doutor em Administração pelo Centro de pós-graduação em Administração (CEPEAD/UFMG). Atua como professor do programa de pós-graduação em Administração da Pontifícia Universidade Católica de Minas Gerais (PUC/MG). Contato:marcrez@hotmail.com 